\title{
A Novel 3D Joint Markov-Gibbs Model for Extracting Blood Vessels from PC-MRA Images
}

\author{
Ayman El-Baz ${ }^{1}$, Georgy Gimel'farb ${ }^{2}$, Robert Falk ${ }^{3}$, Mohamed Abou El-Ghar ${ }^{4}$, \\ Vedant Kumar ${ }^{1}$, and David Heredia ${ }^{1}$ \\ 1 Bioimaging Laboratory, University of Louisville, Louisville, KY, USA \\ 2 Department of Computer Science, University of Auckland, Auckland, New Zealand \\ 3 Director, Medical Imaging Division, Jewish Hospital, Louisville, KY, USA \\ 4 Urology and Nephrology Department, University of Mansoura, Mansoura, Egypt
}

\begin{abstract}
New techniques for more accurate segmentation of a 3D cerebrovascular system from phase contrast (PC) magnetic resonance angiography (MRA) data are proposed. In this paper, we describe PC-MRA images and desired maps of regions by a joint Markov-Gibbs random field model (MGRF) of independent image signals and interdependent region labels but focus on most accurate model identification. To better specify region borders, each empirical distribution of signals is precisely approximated by a Linear Combination of Discrete Gaussians (LCDG) with positive and negative components. We modified the conventional Expectation-Maximization (EM) algorithm to deal with the LCDG. The initial segmentation based on the LCDG-models is then iteratively refined using a MGRF model with analytically estimated potentials. Experiments with both the phantoms and real data sets confirm high accuracy of the proposed approach.
\end{abstract}

\section{Introduction}

Accurate cerebrovascular segmentation using non-invasive MRA is a valuable tool for early diagnostics and timely treatment of intracranial vascular diseases. Among three common MRA techniques, such as time-of-flight MRA (TOFMRA), phase contrast angiography (PCA), and contrast enhanced MRA (CEMRA), only TOF-MRA and PCA use flowing blood as an inherent contrast medium, while for CE-MRA a contrasting substance has to be injected into the circulatory system. Our work is motivated by the wide use of PCA and TOFMRA in clinical practice.

Today's most popular techniques for segmenting blood vessels from MRA data can be roughly classified in two categories: deformable models and statistical methods. The former iteratively adjust an initial boundary surface to blood vessels by optimizing an energy function that depends on image gradient and surface smoothness 1]. Topologically adaptable surfaces make classical deformable models more efficient in segmenting intracranial vasculature [2]. Geodesic active contours implemented with level set techniques offer flexible topological adaptability to segment MRA images [3] including more efficient adaptation to local 
geometric structures represented e.g. by tensor eigenvalues [4]. Fast segmentation of blood vessel surfaces is obtained by inflating a 3D balloon with fast marching methods [5]. In 6] they used a marked point-based segmentation algorithm to extract the coronary tree from 2D X-ray angiography.

The latter extract the vascular tree automatically, but their accuracy depends on underlying probability models. The MRA images are multi-modal in the sense that particular modes of the marginal probability distribution of signals are associated with regions-of-interest. To the best of our knowledge, the only adaptive statistical approaches for extracting blood vessels from the MRA data were proposed by Noble and her group [7/8. The marginal distribution is modeled with a mixture of two Gaussian and one uniform or Rician components for the stationary CSF and bones, brain tissues, and arteries, respectively. The uniform component presumes the blood flow is strictly laminar. In [9] they presented a segmentation algorithm to extract the vascular system from TOF-MRA images. Their approach is based on using a mixture of Gaussian and Rayleigh distributions to approximate the normalized histogram of TOF-MRA images.

\section{Joint Markov-Gibbs Model of PC-MRA Images}

Let $\mathbf{R}=\{(i, j, z): 1 \leq i \leq I, 1 \leq j \leq J, 1 \leq z \leq Z\}$ denote a finite arithmetic grid supporting grayscale $\mathrm{PC}-\mathrm{MRA}$ images $\mathbf{g}: \mathbf{R} \rightarrow \mathbf{Q}$ and their region maps $\mathbf{m}: \mathbf{R} \rightarrow \mathbf{X}$. Here, $\mathbf{Q}=\{0, \ldots, Q-1\}$ and $\mathbf{X}=\{1, \ldots, X\}$ are the sets of gray levels and region labels, respectively, where $Q$ is the number of gray levels and $X$ is the number of image classes. The MGRF model of images to segment is given by a joint probability distribution of PC-MRA images and desired region maps $P(\mathbf{g}, \mathbf{m})=P(\mathbf{m}) P(\mathbf{g} \mid \mathbf{m})$. Here, $P(\mathbf{m})$ is an unconditional distribution of maps and $P(\mathbf{g} \mid \mathbf{m})$ is a conditional distribution of images, given a map. The Bayesian MAP estimate of the map, given the image $\mathbf{g}, \mathbf{m}^{*}=\arg \max _{\mathbf{m}} L(\mathbf{g}, \mathbf{m})$ maximizes the log-likelihood function:

$$
L(\mathbf{g}, \mathbf{m})=\log P(\mathbf{g} \mid \mathbf{m})+\log P(\mathbf{m})
$$

\subsection{Spatial Interaction Model of PC-MRA Images}

Generic Markov-Gibbs model of region maps that accounts for only pairwise interactions between each region label and its neighbors has generally an arbitrary interaction structure and arbitrary Gibbs potentials identified from image data. For simplicity, we restrict the interactions to the nearest voxels (26-neighborhood) and assume, by symmetry considerations, that the interactions are independent of relative region orientation, are the same for all classes, and depend only on intra- or inter-region position of each voxel pair (i.e. whether the labels are equal or not). Under these restrictions, the model is similar to the conventional auto-binomial ones and differs only in that the potentials are not related to a predefined function and have analytical estimates. The symmetric label interactions are three-fold: the closest horizontal-vertical-diagonal in the 
current slice (hvdc), the closest horizontal-vertical-diagonal in the upper slice (hvdu), and the closest horizontal-vertical-diagonal in the lower slice (hvdl). The potentials of each type are bi-valued because only coincidence or difference of the labels are taken into account. Let $\mathbf{V}_{a}=\left\{V_{a}(x, \chi)=V_{a, \text { eq }}\right.$ if $x=\chi$ and $V_{a}(x, \chi)=V_{a, \text { ne }}$ if $\left.x \neq \chi: x, \chi \in \mathbf{X}\right\}$ denote bi-valued Gibbs potentials describing symmetric pairwise interactions of type $a \in \mathbf{A}=\{\mathrm{hvdc}$, hvdu, hvdl $\}$ between the region labels. Let $\mathbf{N}_{\text {hvdc }}=\{(1,0,0),(0,1,0),(-1,0,0),(0,-1,0)\}, \mathbf{N}_{\text {hvdu }}=$ $\{(0,0,1),(-1,-1,1),(-1,1,1),(1,-1,1),(1,1,1)\}$, and $\mathbf{N}_{\text {hvdl }}=\{(0,0,-1),(-1$, $-1,-1),(-1,1,-1),(1,-1,-1),(1,1,-1)\}$ be subsets of inter-voxel offsets for the 26-neighborhood system. Then the Gibbs probability distribution of region maps is:

$$
P(\mathbf{m}) \propto \exp \left(\sum_{(i, j, z) \in \mathbf{R}} \sum_{a \in \mathbf{A}} \sum_{(\xi, \eta, \zeta) \in \mathbf{N}_{a}} V_{a}\left(m_{i, j, z}, m_{i+\xi, j+\eta, z+\zeta}\right)\right)
$$

To identify the MGRF model described in Eq. (2), we have to estimate the Gibbs Potentials V. In this paper we introduce a new analytical maximum likelihood estimation for the Gibbs potentials.

$$
V_{a, \mathrm{eq}}=\frac{X^{2}}{X-1}\left(f_{a}^{\prime}(\mathbf{m})-\frac{1}{X}\right) \text { and } V_{a, \mathrm{ne}}=\frac{X^{2}}{X-1}\left(f_{a}^{\prime \prime}(\mathbf{m})-1+\frac{1}{X}\right)
$$

where $f_{a}^{\prime}(\mathbf{m})$ and $f_{a}^{\prime \prime}(\mathbf{m})$ denote the relative frequency of the equal and non-equal pairs of the labels in all the equivalent voxel pairs $\{((i, j, z),(i+\xi, j+\eta, z+\zeta))$ : $\left.(i, j, z) \in \mathbf{R} . ;(i+\xi, j+\eta, z+\zeta) \in \mathbf{R} ;(\xi, \eta, \zeta) \in \mathbf{N}_{a}\right\}$, respectively.

\subsection{Intensity Model of PC-MRA Images}

Let $q ; q \in \mathbf{Q}=\{0,1, \ldots, Q-1\}$, denote the $Q$-ary gray level. The discrete Gaussian is defined as the probability distribution $\Psi_{\theta}=(\psi(q \mid \theta): q \in \mathbf{Q})$ on $\mathbf{Q}$ such that $\psi(q \mid \theta)=\Phi_{\theta}(q+0.5)-\Phi_{\theta}(q-0.5)$ for $q=1, \ldots, Q-2, \psi(0 \mid \theta)=\Phi_{\theta}(0.5)$, $\psi(Q-1 \mid \theta)=1-\Phi_{\theta}(Q-1.5)$ where $\Phi_{\theta}(q)$ is the cumulative Gaussian function with a shorthand notation $\theta=\left(\mu, \sigma^{2}\right)$ for its mean, $\mu$, and variance, $\sigma^{2}$.

We assume the number $K$ of dominant modes, i.e. regions or classes of interest in a given PC-MRA images, is already known. In contrast to a conventional mixture of Gaussians and/or other simple distributions, one per region, we closely approximate the empirical gray level distribution for PC-MRA images with an LCDG having $C_{\mathrm{p}}$ positive and $C_{\mathrm{n}}$ negative components such that $C_{\mathrm{p}} \geq K$ :

$$
p_{\mathbf{w}, \Theta}(q)=\sum_{r=1}^{C_{\mathrm{p}}} w_{\mathrm{p}, r} \psi\left(q \mid \theta_{\mathrm{p}, r}\right)-\sum_{l=1}^{C_{\mathrm{n}}} w_{\mathrm{n}, l} \psi\left(q \mid \theta_{\mathrm{n}, l}\right)
$$

under the obvious restrictions on the weights $\mathbf{w}=\left[w_{p, .}, w_{n, .}\right]$ : all the weights are non-negative and

$$
\sum_{r=1}^{C_{\mathrm{p}}} w_{\mathrm{p}, r}-\sum_{l=1}^{C_{\mathrm{n}}} w_{\mathrm{n}, l}=1
$$


To identify the LCDG-model including the numbers of its positive and negative components, we modify the EM algorithm to deal with the LCDG.

First, the numbers $C_{\mathrm{p}}-K, C_{\mathrm{n}}$ and parameters $\mathbf{w}, \boldsymbol{\Theta}$ (weights, means, and variances) of the positive and negative Discrete Gaussian (DG) components are estimated with a sequential EM-based initializing algorithm. The goal is to produce a close initial LCDG-approximation of the empirical distribution.

Sequential EM-based Initialization. Sequential EM-based initialization forms an LCDG-approximation of a given empirical marginal gray level distribution using the conventional EM-algorithm [10] adapted to the DGs. At the first stage, the empirical distribution is represented with a mixture of $K$ positive DGs, each dominant mode being roughly approximated with a single DG. At the second stage, deviations of the empirical distribution from the dominant $K$-component mixture are modeled with other, "subordinate" components of the LCDG. The resulting initial LCDG has $K$ dominant weights, say, $w_{\mathrm{p}, 1}, \ldots$, $w_{\mathrm{p}, K}$ such that $\sum_{r=1}^{K} w_{\mathrm{p}, r}=1$, and a number of subordinate weights of smaller values such that $\sum_{r=K+1}^{C_{\mathrm{p}}} w_{\mathrm{p}, r}-\sum_{l=1}^{C_{\mathrm{n}}} w_{\mathrm{n}, l}=0$.

Modified EM Algorithm for LCDG. Modified EM algorithm for LCDG maximizes the log-likelihood of the empirical data by the model parameters assuming statistically independent signals:

$$
L(\mathbf{w}, \boldsymbol{\Theta})=\sum_{q \in \mathbf{Q}} f(q) \log p_{\mathbf{w}, \boldsymbol{\Theta}}(q)
$$

A local maximum of the log-likelihood in Eq. (6) is given with the EM process extending the one in [10] onto alternating signs of the components. Let $p_{\mathbf{w}, \Theta}^{[m]}(q)=$ $\sum_{r=1}^{C_{\mathrm{p}}} w_{\mathrm{p}, r}^{[m]} \psi\left(q \mid \theta_{\mathrm{p}, r}^{[m]}\right)-\sum_{l=1}^{C_{\mathrm{n}}} w_{\mathrm{n}, l}^{[m]} \psi\left(q \mid \theta_{\mathrm{n}, l}^{[m]}\right)$ denote the current LCDG at iteration $m$. Relative contributions of each signal $q \in \mathbf{Q}$ to each positive and negative DG at iteration $m$ are specified by the respective conditional weights

$$
\pi_{\mathrm{p}}^{[m]}(r \mid q)=\frac{w_{\mathrm{p}, r}^{[m]} \psi\left(q \mid \theta_{\mathrm{p}, r}^{[m]}\right)}{p_{\mathbf{w}, \boldsymbol{\Theta}}^{[m]}(q)} ; \quad \pi_{\mathrm{n}}^{[m]}(l \mid q)=\frac{w_{\mathrm{n}, l}^{[m]} \psi\left(q \mid \theta_{\mathrm{n}, l}^{[m]}\right)}{p_{\mathbf{w}, \boldsymbol{\Theta}}^{[m]}(q)}
$$

such that the following constraints hold:

$$
\sum_{r=1}^{C_{\mathrm{p}}} \pi_{\mathrm{p}}^{[m]}(r \mid q)-\sum_{l=1}^{C_{\mathrm{n}}} \pi_{\mathrm{n}}^{[m]}(l \mid q)=1 ; q=0, \ldots, Q-1
$$

The following two steps iterate until the log-likelihood changes become small:

E- step $^{[m+1]}$ : Find the weights of Eq. (17) under the fixed parameters $\mathbf{w}^{[m]}$, $\Theta^{[m]}$ from the previous iteration $m$, and

$\mathbf{M}-\mathbf{s t e p}^{[m+1]}$ : Find conditional MLEs $\mathbf{w}^{[m+1]}, \boldsymbol{\Theta}^{[m+1]}$ by maximizing $L(\mathbf{w}, \boldsymbol{\Theta})$ under the fixed weights of Eq. (7). 
Considerations closely similar to those in [10 show this process converges to a local log-likelihood maximum. Let the log-likelihood of Eq. (6) be rewritten in the equivalent form with the constraints of Eq. (8) as unit factors:

$$
L\left(\mathbf{w}^{[m]}, \mathbf{\Theta}^{[m]}\right)=\sum_{q=0}^{Q} f(q)\left[\sum_{r=1}^{C_{\mathrm{p}}} \pi_{\mathrm{p}}^{[m]}(r \mid q) \log p^{[m]}(q)-\sum_{l=1}^{C_{\mathrm{n}}} \pi_{\mathrm{n}}^{[m]}(l \mid q) \log p^{[m]}(q)\right]
$$

Let the terms $\log p^{[m]}(q)$ in the first and second brackets be replaced with the equal terms $\log w_{\mathrm{p}, r}^{[m]}+\log \psi\left(q \mid \theta_{\mathrm{p}, r}^{[m]}\right)-\log \pi_{\mathrm{p}}^{[m]}(r \mid q)$ and $\log w_{\mathrm{n}, l}^{[m]}+\log \psi\left(q \mid \theta_{\mathrm{n}, l}^{[m]}\right)-$ $\log \pi_{\mathrm{n}}^{[m]}(l \mid q)$, respectively, which follow from Eq. (7). At the E-step, the conditional Lagrange maximization of the log-likelihood of Eq. (9) under the $Q$ restrictions of Eq. (8) results just in the weights $\pi_{\mathrm{p}}^{[m+1]}(r \mid q)$ and $\pi_{\mathrm{n}}^{[m+1]}(l \mid q)$ of Eq. (7) for all $r=1, \ldots, C_{\mathrm{p}} ; l=1, \ldots, C_{\mathrm{n}}$ and $q \in \mathbf{Q}$. At the M-step, the DG weights $w_{\mathrm{p}, r}^{[m+1]}=\sum_{q \in \mathbf{Q}} f(q) \pi_{\mathrm{p}}^{[m+1]}(r \mid q)$ and $w_{\mathrm{n}, l}^{[m+1]}=\sum_{q \in \mathbf{Q}} f(q) \pi_{\mathrm{n}}^{[m+1]}(l \mid q)$ follow from the conditional Lagrange maximization of the log-likelihood in Eq. (9) under the restriction of Eq. (5) and the fixed conditional weights of Eq. (77). Under these latter, the conventional MLEs of the parameters of each DG stem from maximizing the log-likelihood after each difference of the cumulative Gaussians is replaced with its close approximation with the Gaussian density (below "c" stands for "p" or "n", respectively):

$$
\begin{aligned}
& \mu_{\mathrm{c}, r}^{[m+1]}=\frac{1}{w_{\mathrm{c}, r}^{[m+1}} \sum_{q \in \mathbf{Q}} q \cdot f(q) \pi_{\mathrm{c}}^{[m+1]}(r \mid q) \\
& \left(\sigma_{\mathrm{c}, r}^{[m+1]}\right)^{2}=\frac{1}{w_{\mathrm{c}, r}^{[m+1}} \sum_{q \in \mathbf{Q}}\left(q-\mu_{\mathrm{c}, i}^{[m+1]}\right)^{2} \cdot f(q) \pi_{\mathrm{c}}^{[m+1]}(r \mid q)
\end{aligned}
$$

This modified EM-algorithm is valid until the weights $\mathbf{w}$ are strictly positive. The iterations should be terminated when the log-likelihood of Eq. (6) does not change or begins to decrease due to accumulation of rounding errors.

The final mixed LCDG-model $p_{C}(q)$ is partitioned into the $K$ LCDG-submodels $P_{[k]}=[p(q \mid k): q \in \mathbf{Q}]$, one per class $k=1, \ldots, K$, by associating the subordinate DGs with the dominant terms so that the misclassification rate is minimal.

\section{Experimental Results}

Experiments were conducted with the PC-MRA images acquired with the Picker 1.5T Edge MRI scanner having spatial resolution of $0.86 \times 0.86 \times 1.0 \mathrm{~mm}$. The size of each $3 \mathrm{D}$ data set is $256 \times 256 \times 123$. The PC-MRA images contain three classes $(K=3)$, namely, darker bones and fat, brain tissues, and brighter blood vessels. A typical PC-MRA slice, its empirical marginal gray level distribution $f(q)$, and the initial 3-component Gaussian dominant mixture $p_{3}(q)$ are shown in Fig. 1 .

Figure 2 presents the final LCDG-model after refining the initial one with the modified EM-algorithm and shows successive changes of the log-likelihood at the refinement iterations. The final LCDG-models of each class are obtained with 
Table 1. Minimum $\varepsilon_{\mathrm{n}}$, maximum $\varepsilon_{\mathrm{x}}$, and mean $\bar{\varepsilon}$ segmentation errors, and standard deviations $\sigma$ of errors on the geometrical 3D PC-MRA phantoms for our (OA) as well as for four other segmentation algorithms using iterative thresholding (IT) [11, gradient based (DMG) 12] or gradient vector flow based (GVF) [13 deformable models, and Chung segmentation approach $(\mathrm{C}) 8$

\begin{tabular}{|l|c|c|c|c|c|}
\cline { 2 - 6 } \multicolumn{1}{c|}{} & OA & IT & DMG & GVF & C \\
\hline$\varepsilon_{\mathrm{n}}, \%$ & $\mathbf{0 . 0 7}$ & 3.89 & 8.9 & 1.97 & 0.1 \\
$\varepsilon_{\mathrm{x}}, \%$ & $\mathbf{1 . 8 7}$ & 31.7 & 19.1 & 11.1 & 12.1 \\
$\bar{\varepsilon}, \%$ & $\mathbf{0 . 4 9}$ & 15.7 & 9.8 & 4.87 & 6.2 \\
$\sigma, \%$ & $\mathbf{0 . 8 1}$ & 7.01 & 2.99 & 1.79 & 0.93 \\
\hline
\end{tabular}

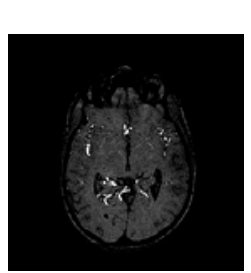

(a)

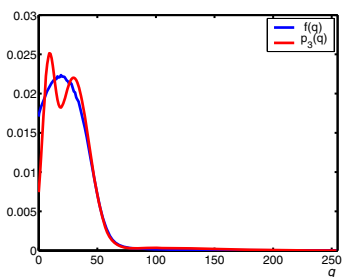

(b)

Fig. 1. Typical PC-MRA scan slice (a) and deviations between the empirical distribution $f(q)$ and the dominant 3component mixture $p_{3}(q)$ (b)

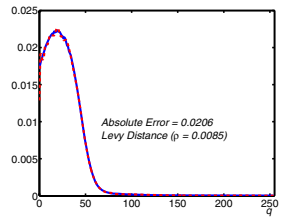

(a)

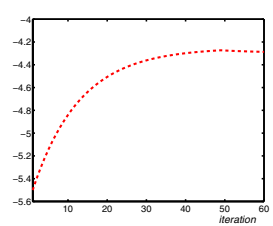

(b)

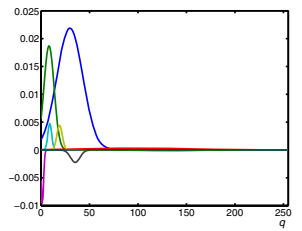

(c)

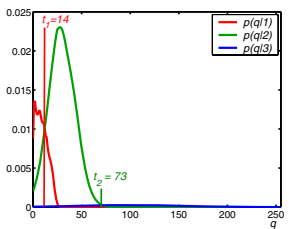

(d)

Fig. 2. Final 3-class LCDG-model overlaying the empirical density (a), the loglikelihood dynamics (b) for the refining EM-iterations, the refined model components (c), and the class LCDG-models (d)

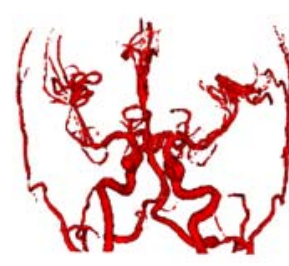

(a)

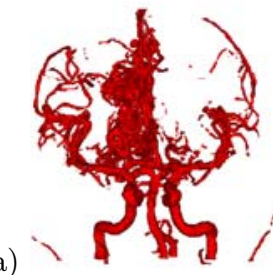

(b)

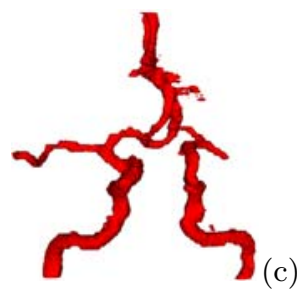

(c)

Fig. 3. Segmentation results obtained by the proposed approach

the best separation thresholds $t_{1}=14$ and $t_{2}=73$. The first 47 refining iterations increase the log-likelihood from -5.5 to -4.27 . It is clear from Fig. 2(a) that the LCDG helped us to better approximate the tails of the empirical density as well as its main body. Better approximation will lead to good initial segmentation.

The region map obtained first with only the class LCDG-models is further refined using the iterative segmentation algorithm. Changes in the likelihood $L(\mathbf{g}, \mathbf{m})$ become very small after 9 iterations. For this map the initial estimated 
parameters are $V_{a, e q}=-V_{a, n e}=1.71$, and the final estimated parameters are $V_{a, e q}=-V_{a, n e}=2.13$. The final region map produced with these parameters using the Metropolis voxelwise relaxation is shown in Fig. 3(a). More segmentation results are shown in Figs 3 (b,c).

\section{Validation and Conclusions}

It is very difficult to get accurate manually segmented complete vasculare trees to validate our algorithm. To quantitatively evaluate its performance, we created three wooden 3D phantoms in Fig. 4 with geometrical shapes similar to blood vessels. They mimic bifurcations and zero and high curvature existing in any vascular system, and their changing radii simulate both large and small blood vessels. The scanned phantoms were manually segmented to obtain the ground truth. The blood vessel and non-vessel signals for each phantom were generated according to the class distributions $p(q \mid 1), p(q \mid 2)$, and $p(q \mid 3)$ in Fig. $2(\mathrm{~d})$ using the inverse mapping methods. The resulting phantom's histograms are similar to that in Fig. 2(a).

The total segmentation error is evaluated by a percentage of erroneous voxels with respect to the overall number of voxels in the manually segmented 3D phantom. Figure 4 shows the segmentation of the three phantoms using our approach. Table 1 gives error statistics for 440 synthetic slices segmented in the phantoms with proposed approach and compares them to four other known segmentation algorithms. The statistical analysis using a two tailed t-test shows that there is a significant difference $\left(P<10^{-4}\right)$ between the error generated by our segmentation approach and the error generated by the other four algorithms that are cited in Table 1 which highlight the advantages of the proposed approach. Figure 4 compares the results of our segmentation approach and the Chung-Noble's segmentation approach, the errors being in terms of the number

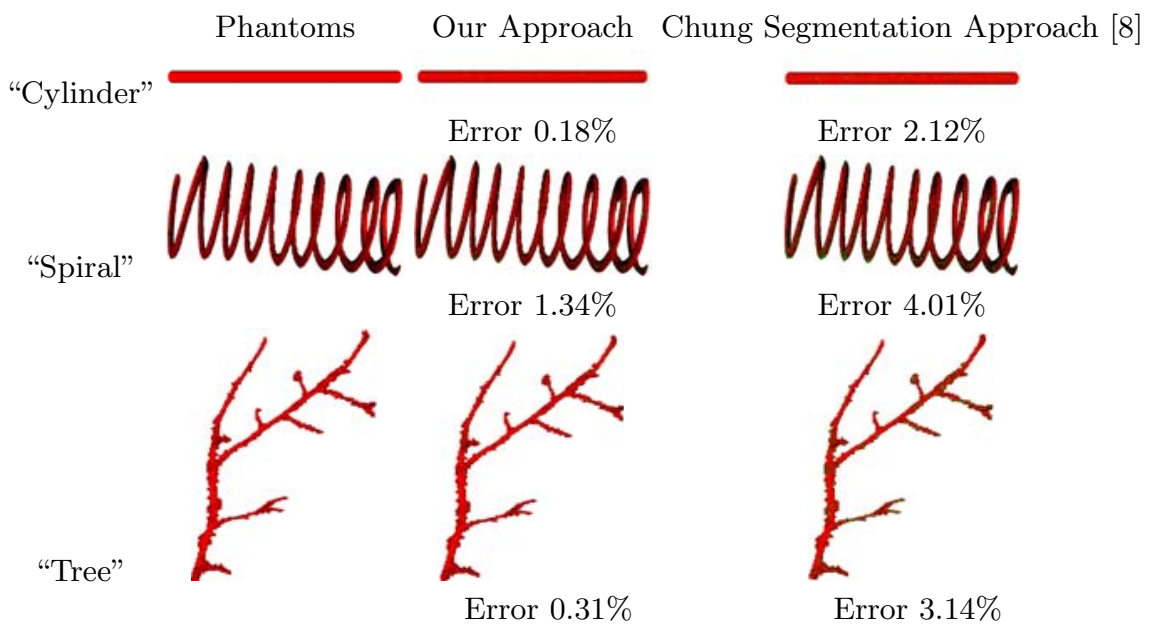

Fig. 4. Segmentation of 3D phantoms. Error shown in green. 
of wrong (i.e. missed or extra) voxels relative to the total voxel number in the manually segmented 3D phantoms. In total, our approach produces $0.18-1.34 \%$ erroneous voxels comparing to $2.12-4.01 \%$ for the Chung-Noble's approach on the synthetic PC-MRA data.

We presented a new stochastic approach to find blood vessels in multi-modal PC-MRA images. The LCDG-model accurately approximates the empirical marginal gray level distribution yielding the high quality segmentation. The accuracy of our approach is validated using a specially designed 3D geometrical phantom. LCDG-model ensures fast convergence of the model refinement with the modified EM algorithm. Also, we introduced a new analytical method for accurate estimation of 3D auto-binomial MGRF model. The proposed approach is not limited only for PC-MRA but also is suitable for segmenting TOF-MRA and CTA medical images. The latter were not included in the paper because of the space limitations, but, the algorithm's code, sample data and segmentation results for the TOF-MRA, PC-MRA, and CTA images will be provided in our web site.

\section{References}

1. Caselles, V., Kimmel, R., Sapiro, G.: Geodesic active contours. Int. J. Computer Vision 22, 61-79 (1997)

2. McInerney, T., Terzopoulos, D.: Medical image segmentation using topologically adaptable surface. In: Troccaz, J., Mösges, R., Grimson, W.E.L. (eds.) CVRMedMRCAS 1997, CVRMed 1997, and MRCAS 1997. LNCS, vol. 1205, pp. 23-32. Springer, Heidelberg (1997)

3. Lorigo, L., Faugeras, O., Grimson, W.E.L., Keriven, R.: Curves: Curve evolution for vessel segmentation. Medical Image Analysis 5, 195-206 (2001)

4. Wink, O., Niessen, W.J., Viergever, M.A.: Fast delineation and visualization of vessels in 3-D angiographic images. IEEE TMI 19, 337-346 (2000)

5. Deschamps, T., Cohen, L.D.: Fast extraction of tubular and tree 3D surfaces with front propoagation methods. In: Proc. $16^{\text {th }}$ ICPR, pp. 731-734 (2002)

6. Lacoste, C., Finet, G., Magnin, I.: Coronary tree extraction from X-ray angiograms using marked point processes. In: Proc. of IEEE International Symposium on Biomedical Imaging: From Nano to Macro (ISBI 2006), Arlington, Virginia, USA, April 6-9, pp. 157-160 (2006)

7. Wilson, D.L., Noble, J.A.: An adaptive segmentation algorithm for time-of-flight MRA data. IEEE Trans. Med. Imaging 18, 938-945 (1999)

8. Chung, A., Noble, J.A., Summers, P.: Fusing speed and phase information for vascular segmentation of phase contrast MR angiograms. MIA 6, 109-128 (2002)

9. Hassouna, M., Farag, A., Hushek, S., Moriarty, T.: Cerebrovascular Segmentation from TOF Using Stochastic Models. Medical Image Analysis 10(1), 2-16 (2006)

10. Schlesinger, M., Hlavac, V.: Ten Lectures on Statistical and Structural Pattern Recognition. Kluwer Academic, Dordrecht (2002)

11. $\mathrm{Hu}, \mathrm{S}$., Hoffman, E.A.: Automatic lung segmentation for accurate quantization of volumetric X-ray CT images. IEEE TMI 20, 490-498 (2001)

12. Kass, M., Witkin, A., Terzopoulos, D.: Snakes: Active contour models. Int. J. Computer Vision 1, 321-331 (1987)

13. Xu, C., Prince, J.L.: Snakes, shapes, and gradient vector flow. IEEE TIP 7, 359-369 (1998) 